\title{
MAGNETICALLY INDUCED PHASE CONDENSATION WITH ASYMPTOTIC CRITICAL TEMPERATURE IN AN AQUEOUS MAGNETIC COLLOID
}

\author{
V.Socoliuc ${ }^{1,2 *}$, D. Bica ${ }^{2}$, L. Vékás \\ 1 "Petru Poni" Institute of Macromolecular Chemistry, \\ $41 A$ Grigore Ghica Voda Alley, 700487 Iasi, Romania \\ ${ }^{2}$ Laboratory of Magnetic Fluids, Center for Fundamental and Advanced Technical \\ Research, Romanian Academy - Timisoara Branch, \\ Bv. M. Viteazu 24, Timisoara RO-300223, Romania \\ *e-Mail: vsocoliuc@acad-tim.tm.edu.ro
}

\begin{abstract}
We investigated the phenomenon of magnetically induced phase condensation in a water-based magnetic nanocolloid with magnetite nanoparticles of $5 \mathrm{~nm}$ in diameter, sterically stabilized by a double surfactant layer of myristic acid (MA) and dodecylbenzenesulphonic acid (DBS). It is found that the sample presents an asymptotic critical temperature $T_{\infty} \approx 40^{\circ} \mathrm{C}$, above which no condensation occurs, no matter how intense the external magnetic field is. Below the asymptotic critical temperature there is a temperature dependent critical field $H_{\mathrm{c}}(T)$, below which no condensation occurs and above which the amount and the size (in the micron range) of the condensed phase drops increase with the field intensity. The critical field exponentially increases as the temperature approaches $T_{\infty}$. From DLS investigations it was found that the magnetic nanoparticles spontaneously agglomerated, most likely due to van der Waals and/or hydrophobic interactions, into clusters whose population diminished with the increasing temperature.
\end{abstract}

Introduction. Phase condensation is among the most important structuring phenomena in magnetic nanocolloids (a.k.a. ferrofluids, magnetic fluids or magnetic nanofluids) that is important from both fundamental and technological points of view. As a result of the phase condensation, which can be spontaneous, but most often magnetically induced, the magnetic nanocolloid separates into two phases at equilibrium [1-4]. The condensed phase drops, containing highly packed magnetic nanoparticles, are suspended in the matrix of the diluted phase. In the presence of an external magnetic field, the condensed phase drops are prolate ellipsoids aligned in the field direction with lengths that can range up to several tens of microns and thicknesses that can range from several hundreds of nanometers to several microns $[5,6]$. The colloidal stability of magnetic nanofluids with respect to the phase separation phenomenon depends on a variety of factors like the magnetic nanoparticle concentration, size and size polydispersity, the colloidal stabilization efficiency, temperature and magnetic field intensity.

In this paper, we investigate the magnetically induced phase condensation phenomenon in an aqueous magnetic nanocolloid, where spontaneous particle clustering occurs due to van der Waals and/or hydrophobic interactions. The influence of the temperature both on the spontaneous clustering and on the magnetically induced condensation is studied.

1. Experimental. A water-based magnetic nanocolloid with magnetite nanoparticles was produced by means of the chemical coprecipitation method [7]. The nanoparticles were sterically stabilized by a double surfactant layer of myristic acid 
(MA), chemically adsorbed on the particle surface, and dodecyl-benzenesulphonic acid (DBS), physically adsorbed on the MA layer. The sample's magnetic particle volume fraction was $2.3 \%$. The particles' size statistic was determined from TEM investigations.

A Malvern Zetasizer Nano ZS instrument was used for Dynamic Light Scattering (DLS) investigations of the sample's structure in the temperature range $20-90^{\circ} \mathrm{C}$ and in the absence of external magnetic field.

The sample's structure under the influence of an external magnetic field in the temperature range $20-70^{\circ} \mathrm{C}$ was investigated by means of light extinction experiments, using an in-house built optical bench [6]. Due to scattering on the condensed phase drops, the light transmitted through the sample in the forward direction, perpendicular to the magnetic field, is the more extinct the larger the size and the number of the condensed phase drops. Thus, the extinction expressed as

$$
\operatorname{Ext}(H)=\frac{I(H=0)-I(H)}{I(H=0)},
$$

measures the extent of the magnetically induced phase condensation in the sample [6]. $I(H)$ is the laser (He-Ne, $\lambda=632 \mathrm{~nm}$ ) light intensity emergent from the sample in the forward direction, after the condensation process reached equilibrium as the result of the sudden onset of the magnetic field of intensity $H$.

2. Results and discussion. From the TEM analysis, the physical diameter of magnetite nanoparticles was sampled. The physical diameter of the magnetite particles in the sample was found to be $D_{\mathrm{p}}=5.1 \pm 1.1 \mathrm{~nm}$ [7]. With an estimate of the surfactant layer thickness of $2 \mathrm{~nm}$, the mean hydrodynamic diameter of the coated single particles is about $9 \mathrm{~nm}$.

Fig. 1 illustrates the temperature dependence of the mean hydrodynamic diameter as determined from the cumulants analysis of the DLS intensity autocorrelation function [8]. The mean hydrodynamic diameter monotonously decreases from $47 \mathrm{~nm}$ at $20^{\circ} \mathrm{C}$ to $25 \mathrm{~nm}$ at $90^{\circ} \mathrm{C}$. The highest rate of diameter decrease is within $30^{\circ} \mathrm{C}$ and $60^{\circ} \mathrm{C}$. Compared to the estimated mean hydrodynamic diameter of the coated single particles of about $9 \mathrm{~nm}$, the DLS hydrodynamic diameter indicates that the colloidal particles from the structure of the sample are actually

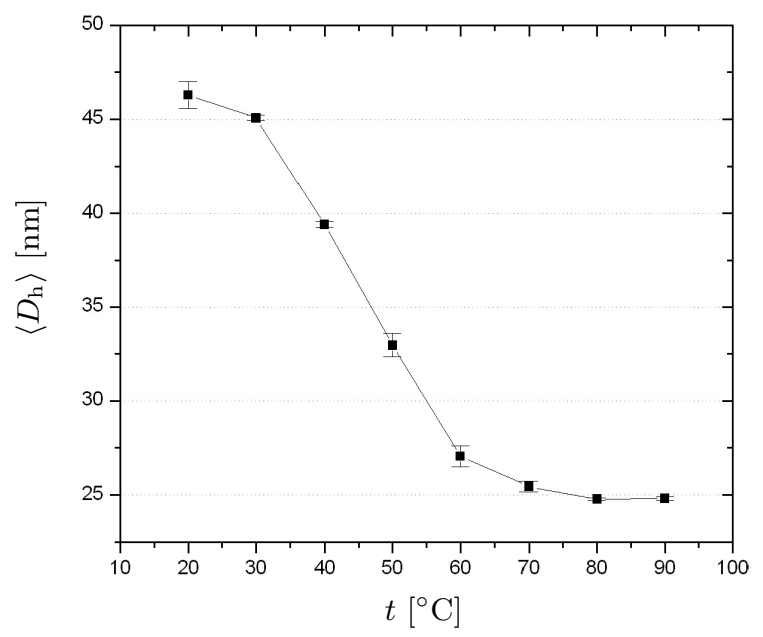

Fig. 1. Temperature dependence of the mean hydrodynamic diameter. 
Magnetically induced phase condensation with asimptotic critical temperature
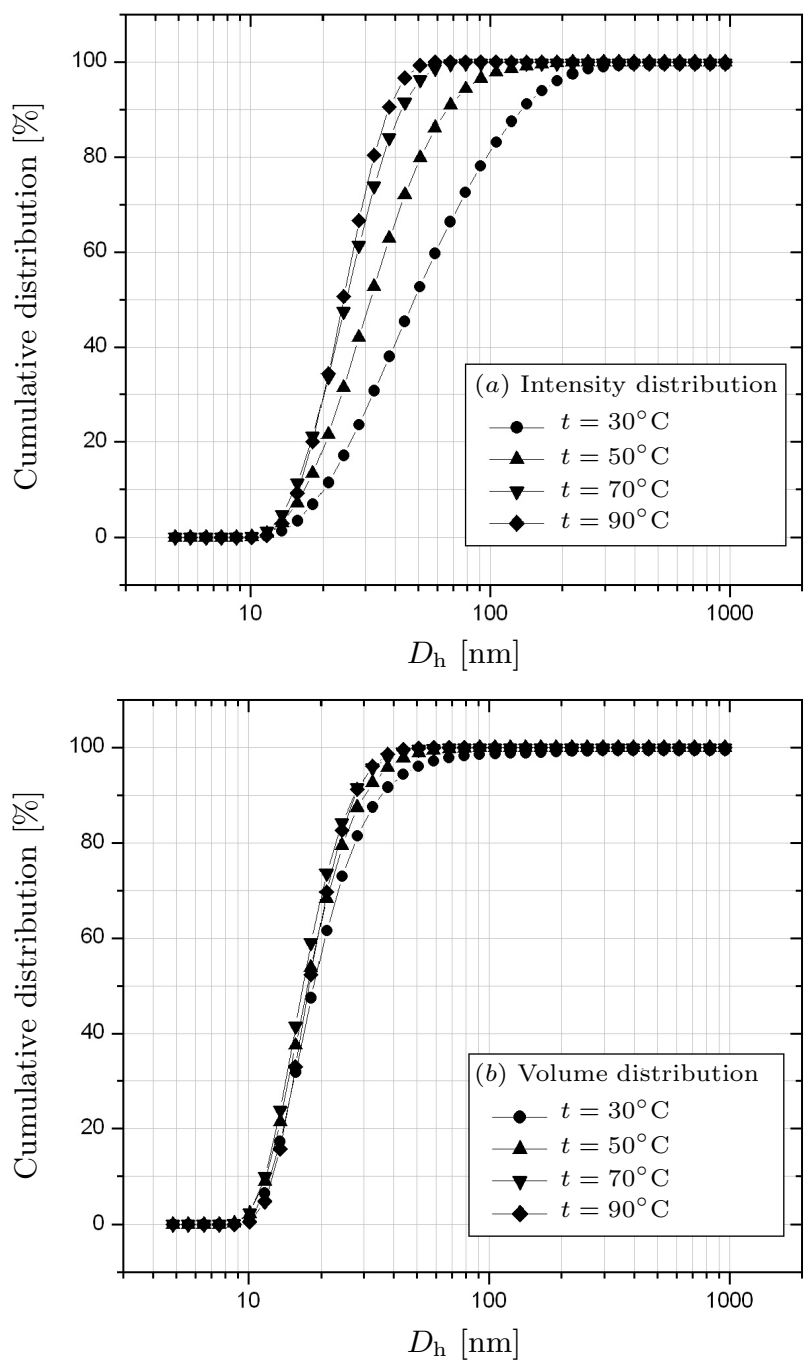

Fig. 2. DLS cumulative distributions by: $(a)$ intensity $\sim D_{\mathrm{h}}^{6}$, and $(b)$ volume $\sim D_{\mathrm{h}}^{3}$.

clusters of magnetite nanoparticles. From the sensitivity of their size to the temperature change, one can infer that the clusters are most likely due to van der Waals and/or hydrophobic interactions. For the $5 \mathrm{~nm}$ mean diameter magnetite particles, the magnetic dipole-dipole interaction is exceeded by the thermal energy.

Fig. 2 shows the DLS cumulative distributions by the scattered intensity and particle cluster volume, respectively, for four temperature values: $30,50,70$ and $90^{\circ} \mathrm{C}$. Because the scattered light intensity depends on the scaterer's squared volume, the size distribution by intensity acts like a magnifier glass for the population of large clusters. Fig. 2a shows a wide polydispersity of the clusters' diameter and that the increasing temperature diminishes the polydispersity by dissolving the largest clusters. From the volume distributions one can estimate the volume fraction of the large clusters. As an example, the clusters with the hydrodynamic diameter larger than $40 \mathrm{~nm}$ sum up to about $8 \%$ at $30^{\circ} \mathrm{C}$ and up to only about $1 \%$ at $70^{\circ} \mathrm{C}$, from the total magnetite nanoparticle content of the sample.

The optical extinction was measured for several temperatures ranging $20^{\circ} \mathrm{C}$ to $70^{\circ} \mathrm{C}$, and for several values of the magnetic field intensity in the range $0-240 \mathrm{kA} / \mathrm{m}$. After the temperature was stabilized, the magnetic field was suddenly turned on 

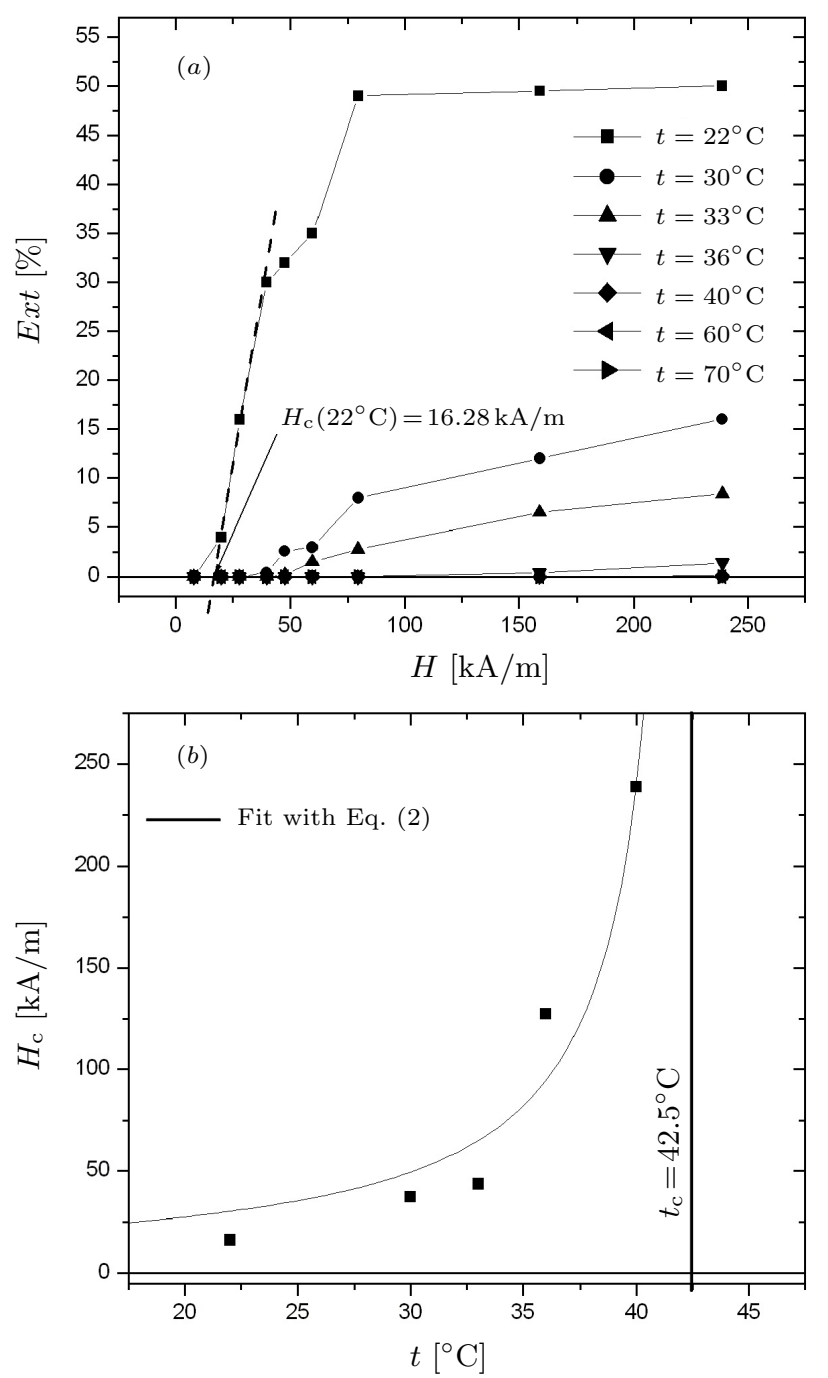

Fig. 3. (a) The magnetic field intensity dependence of extinction at several values of the temperature, and $(b)$ the temperature dependence of the critical field intensity and the fit with Eq. (2).

at the preset intensity value. As the condensation process evolved towards equilibrium, the extinction increased from zero to a saturation value (for details about the kinetics of the magnetically induced phase condensation see [6]). Fig. $3 a$ displays the magnetic field intensity dependence of the saturation extinction at several temperature values. Below $8 \mathrm{kA} / \mathrm{m}$, no magnetic induced particle clustering takes place, i.e. $\operatorname{Ext}(H)=0$ over the entire temperature interval. Above $8 \mathrm{kA} / \mathrm{m}$, the clustering increases with field intensity, but at a constant field the clustering sharply diminishes with the increasing temperature. For each temperature, there is a critical field intensity value $H_{\mathrm{c}}(T)$, below which no condensation occurs. Similarly, at each field intensity $H$ there is a critical temperature $T_{\mathrm{c}}(H)$, above which no condensation occurs. Fig. $3 a$ shows that $\operatorname{Ext}(H)=0$ above $40^{\circ} \mathrm{C}$, thus indicating the existence of a finite asymptotic critical temperature $T_{\infty}$, above which no condensation occurs, no matter how intense the external magnetic field is (the asymptotic critical temperature can be viewed as a limit of $T_{\mathrm{c}}(H)$ when $H$ goes to infinity). This behaviour is typical for the phenomenon of magnetically 
induced phase transition $[1,2]$.

The values of the critical field were determined by linear extrapolation to zero extinction of the first 2-3 nonzero extinction points on the curves $\operatorname{Ext}(\mathrm{H})$ at constant temperature, as exemplified by the dashed line in Fig. $3 a$. The temperature dependence of the critical field intensity $H_{\mathrm{c}}(t)$ is plotted in Fig. $3 b$. One can notice the asymptotic increase of the critical field with the increasing temperature. This temperature dependence of the critical field intensity was theoretically predicted by Cebers [2]:

$$
H_{\mathrm{c}}(T)=\frac{2 k_{B}}{\mu_{0} m} \frac{T_{\infty}^{2}}{T_{\infty}-T}
$$

where $k_{B}$ is the Boltzmann constant, $\mu_{0}$ is the magnetic permeability of vacuum and $T_{\infty}$ is the asymptotic critical temperature. $m$ is the magnetic moment of a magnetite particle

$$
m=\frac{\mu_{0} \pi D_{m}^{3} M_{d}}{6}
$$

where $M_{d}=4.84 \cdot 10^{5} \mathrm{~A} / \mathrm{m}$ is the saturation magnetization of magnetite.

Using Eq. 2 to fit the experimental dependence of the critical field on the temperature from Fig. 3b, one obtains for the asymptotic critical temperature $T_{\infty}=42.5^{\circ} \mathrm{C}$, being very close to a temperature of $40^{\circ} \mathrm{C}$, where we observe no condensation for a maximum available field intensity of $240 \mathrm{kA} / \mathrm{m}$. A numerical value for the first factor in the right-hand side of Eq. 2 was also obtained from the fit

$$
\frac{2 k_{B}}{\mu_{0} m}=6.26 \mathrm{~A} /(\mathrm{mK}) \text {. }
$$

One can use Eq. 3 and Eq. 4 to estimate an effective mean magnetic diameter of the particle clusters: $D_{\mathrm{m}, \mathrm{eff}}=24 \mathrm{~nm}$. Due to the surfactant layer $(2 \mathrm{~nm})$ and to the nonmagnetic layer $(0.85 \mathrm{~nm})$ at the surface of the magnetite nanoparticles, an effective magnetic diameter of $24 \mathrm{~nm}$ leads to an estimate of the cluster hydrodynamic diameter of about $78 \mathrm{~nm}$, which is much larger than the mean hydrodynamic diameter obtained from DLS (Fig. 1). This discrepancy can be attributed to the fact that the Cebers phase transition model assumes identical monodomain particles with a temperature independent diameter. From the DLS cumulative distribution by volume from Fig. $2 b$, the clusters with the hydrodynamic diameter larger than $78 \mathrm{~nm}$ sum up to only $1.8 \%$ at $30^{\circ} \mathrm{C}$ from the total magnetite nanoparticle content of the sample. Nevertheless, the experiments on successively filtered samples carried by Rosensweig and Popplewell [9] showed that the phase condensation could be triggered by only a small amount of large particles present in the structure of a sample. Also, a theoretical evidence that an amount of large particles less than $5 \%$ can be the main drive of the magnetically induced phase instability in bidisperse magnetic nanocolloids was obtained by Ivanov in [3]. On the other hand, the coincidence that the value of the measured asymptotic critical temperature of $40^{\circ} \mathrm{C}$ lays roughly in the middle of the temperature range where DLS shows the steepest decay of the clusters' hydrodynamic diameter with the increasing temperature, raises the question whether the observed phenomenon is a pure phase transition.

3. Conclusions. The experimentally observed magnetically induced phase condensation with asymptotic critical temperature is driven by the small fraction of large particle clusters spontaneously formed due to van der Waals and/or hydrophobic interactions. This is consistent with the predictions of the theoretical model developed by Ivanov [3] for the magnetically induced phase instability in bidisperse magnetic nanocolloids. However, because both the size and the fraction 
of large spontaneous clusters show an abrupt decay with the increasing temperature across the observed asymptotic critical temperature, it is still an open question if the observed phenomenon is solely the result of a pure phase transition.

Acknowledgements. V. Socoliuc and L. Vekas acknowledge the financial support of the European Social Fund - "Cristofor I. Simionescu" Postdoctoral Fellowship Programme (ID POSDRU/89/1.5/S/55216), Sectoral Operational Programme Human Resources Development 2007-2013. This work is dedicated to Dr. Doina Bica (1952-2008) in memory of our long-term scientific cooperation.

\section{REFERENCES}

[1] V. Cabuil, J.C. Bacri, R. Perzynski and Yu. Raikher. Colloidal stability of magnetic fluids. In: Magnetic Fluids and Applications Handbook (Eds. B. Berkovski and V. Bashtovoy, Begell House, New York \& Wallingford (UK), 1996), p. 45.

[2] A. Cebers. Basic physical, mathematical and computer simulation models. In: Magnetic Fluids and Applications Handbook (Eds. B. Berkovski and V. Bashtovoy, Begell House, New York \& Wallingford (UK), 1996), p. 253.

[3] A.O. Ivanov. Phase separation in bidisperse ferrocolloids. J. Magn. Magn. Matter., vol. 154 (1996), p. 66.

[4] A.Yu.Zubarev and L.Yu. Iskakova. Condensation phase transitions in ferrofluids. Physica A, vol. 335 (2004), p. 325.

[5] S. Taketomi, H. Takahsshi, N. Inaba and H. Miyajima. Experimental and theoretical investigations on agglomeration of magnetic colloidal particles in magnetic fluids. J. Phys. Soc. Jpn, vol. 60 (1991), p. 1689.

[6] V. Socoliuc And D. BicA. Experimental investigation of magnetic-induced phase-separation kinetics in aqueous magnetic fluids. Progr. Colloid Polym. Sci., vol. 117 (2001), p. 117.

[7] D. Bica, L. Vekas, M.V. Avdeev, O. Marinica, V. Socoliuc, M. BalaSOIU AND V.M. GARAmus. Sterically stabilized water based magnetic fluids: Synthesis, structure and properties. J. Magn. Magn. Matter., vol. 311 (2007), p. 17.

[8] R. Xu. Particle Characterization: Light Scattering Methods (Kluwer Academic Publishers, New York, Boston, Dordrecht, London, Moscow, 2002), p. 249.

[9] R.E. Rosensweig and J. Popplewell. Influence of concentration on field induced phase transition in magnetic fluids. In: Electromagnetic Forces and Applications. Int. J. Appl. Electromag. Mater. (2 Suppl. 1982), p. 83. 